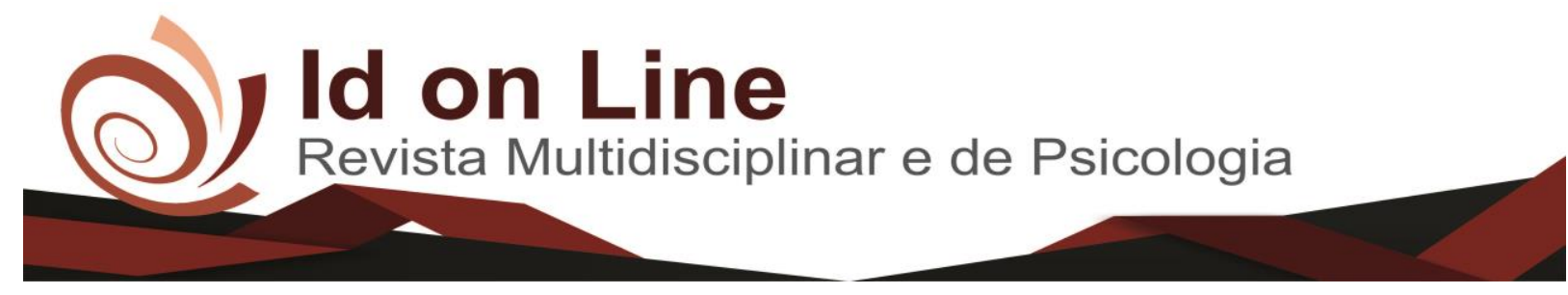

Artigo

\title{
Percepção de mães de recém-nascidos prematuros sobre o cuidado intensivo neonatal
}

Mauro Palmarella Neto ${ }^{\prime}$; Valéria Gomes da Silva²; Laisla Pires Dutra ${ }^{3}$

\begin{abstract}
Resumo: O objetivo do estudo foi conhecer a percepção de mães de recém-nascidos prematuros sobre o cuidado intensivo neonatal. Trata-se de um estudo qualitativo, descritivo e exploratório, realizado com 9 mães de filhos internados em unidades de cuidado intensivo neonatal. As participantes responderam um questionário biosociodemográfico e posteriormente foi solicitado que realizassem um desenho representando seus sentimentos durante o período de internação. Para análise dos dados foi realizado a técnica de análise de conteúdo das histórias temáticas e análise do grafismo. Os resultados foram descritos através da caracterização das mães e a percepção das mesmas sobre o processo de internamento no cuidado intensivo neonatal apresentado em 2 categorias, a primeira: os sentimentos maternos vivenciados durante a hospitalização do filho na unidade de terapia intensiva neonatal, e a segunda: espiritualidade e crenças religiosas. Conclui-se que essa fase do internamento submete as mães a vários sentimentos, seja eles bons e ruins.
\end{abstract}

Palavras chaves: Cuidados intensivos. Mães. Prematuros

\section{Perception of mothers of premature babies on neonatal intensive care}

\begin{abstract}
The aim of the study was to know the perception of mothers of preterm premature babies on neonatal intensive care. This is a qualitative, descriptive and exploratory study carried out with 9 mothers of children hospitalized in neonatal intensive care units. The participants answered a biosociodemographic questionnaire and later were asked to make a drawing representing their feelings during the period of hospitalization. For analysis of the data the technique of analysis of the content of the thematic stories and analysis of the graphism was carried out. The results were described through the characterization of the mothers and their perception of the process of hospitalization in neonatal intensive care presented in 2 categories, the first one: the maternal feelings experienced during the hospitalization of the child in the neonatal intensive care unit, and the second: spirituality and religious beliefs. It is concluded that this phase of hospitalization subjects mothers to various feelings, whether good or bad.
\end{abstract}

Keywords: Intensive care. Mothers. Premature

\footnotetext{
${ }^{1}$ Discente graduando do curso de Fisioterapia pela Faculdade Independente do Nordeste-FAINOR. http:://www.fainor.com.br, Vitória da Conquista-BA, Brasil. mauro.palmarella@ hotmail.com;

${ }^{2}$ Psicoterapeuta, coordenadora de Psicologia da Secretaria de Educação e Cultura, Itiruçu-BA, Pós-graduada em Psicopedagogia pelo Instituto Valdice Cruz-IVAC, Jaguaquara-BA, Brasil. psivgomes@gmail.com;

${ }^{3}$ Fisioterapeuta no Instituto Glass e Mundo Ímpar, Docente da Faculdade Independente do Nordeste-FAINOR, Vitória da Conquista-BA, Mestre em Ciências da Saúde pela Universidade Estadual do Sudoeste da Bahia-UESB, Membro da Associação Brasileira de Fisioterapia Neurofuncional, Vitória da Conquista-BA, Brasil. laysla19@ hotmail.com.
}

Faculdade Independente do Nordeste-FAINOR, Avenida Luís Eduardo Magalhães, número 1035, CEP: 45055-420, Bairro Candeias, Vitória da Conquista-BA. 


\section{Introdução}

Segundo a Organização Mundial de Saúde (OMS), a prematuridade é considerada como um problema mundial de saúde, estando diretamente ligada com o índice de mortalidade neonatal. São considerados prematuros todo nascimento a baixo de 37 semanas de gestação, podendo ser classificado em leve, nascidos de 35 a 37 semanas, moderado, nascidos de 31 a 34 semanas e extremo, nascidos abaixo ou igual a 30 semanas de gestação (OLIVEIRA et al., 2016).

De acordo com Ramos (2009), são pouco conhecidas as causas sobre a prematuridade, mas acredita-se que alguns fatores podem ser responsáveis por um terço dos partos prematuros. Segundo Bettiol (2010), muitos são os fatores que levam ao nascimento de um bebê prematuro, tais eles como: infecções, partos múltiplos, hipertensão induzida pela gravidez, tabagismo e o uso de drogas ilícitas, rotina de trabalho muito intensa, baixo índice de massa corpórea, ganho de peso insuficiente durante o período de gravidez, reprodução assistida, colo uterino curto, intervalo interpartal curto, baixa escolaridade, raça negra e história anterior de nascimento prétermo.

Em consequência dessas condições de nascimentos, seja ela cirúrgica ou clínica, é de extrema importância que os recém-nascidos prematuros recebam os atendimentos na Unidade de Terapia Intensiva Neonatal (UTIN) possibilitando assim um aumento das chances de sobrevivência (CRUZ et al., 2010).

A longa duração do internamento destes bebês nas UTIN impossibilita a ligação e o apego materno, o que favorece situações de insegurança da mãe com os cuidados ao seu(s) filho(s). As perturbações aguçam a vontade de conhecer os sentimentos maternos o que é demonstrada por fios conectados em aparelhos de ventilação mecânica no combate a sobrevivência (RABELO et al., 2007; CRUZ et al., 2010).

O árduo sofrimento psíquico dessas mães diante da prematuridade de seus filhos, pode favorecer o surgimento de perturbações de ansiedade, fobias, e até mesmo de quadros depressivos diante que há uma ameaça de agravos durante o internamento intensivo. Esses sentimentos acompanham as mães desde o momento da idealização de seus filhos, assim como na tão esperada alta hospitalar (ANJOS et al., 2012).

O momento da alta hospitalar é aguardado com bastante ansiedade aos familiares, especialmente para aqueles que irão receber esse bebê em seu meio, visto que, o mesmo precisará de um cuidado e atenção redobrada aos cuidados da mãe (RABELO et al.,2007). 
Compreendendo a importância da mãe no cuidado e acompanhamento do recém-nascido prematuro durante o internamento na unidade de cuidados intensivos neonatais, este estudo teve como objetivo conhecer a percepção de mães de recém-nascidos prematuros sobre o cuidado intensivo neonatal.

\section{Método}

Trata-se de um estudo qualitativo, descritivo e exploratório, realizado nas unidades de Terapia Intensiva Neonatal do Hospital Municipal Esaú Matos (HMEM) e Hospital Geral de Vitória da Conquista (HGVC) localizados no município de Vitória da Conquista - Bahia, considerada a terceira maior cidade do estado da Bahia e do interior do Nordeste, com uma população estimada de 346.069 habitantes no ano de 2016, segundo IBGE. O HGVC é um hospital do estado que assiste à população de Vitória da Conquista e mais de 68 municípios pactuantes do estado da Bahia, com referência em cirurgias e traumato-ortopedia. A unidade de Terapia Intensiva Neonatal do HGVC está em funcionamento desde abril de 2014 e consta de 10 leitos intensivos na unidade. O HMEM é referência em alto risco neonatal, possui 10 leitos de cuidados intensivos e 24 leitos de cuidados intermediários neonatal.

A amostra do estudo foi composta por 9 mães de recém-nascidos prematuros, que tiveram seus filhos internados na Unidade de Terapia Intensiva Neonatal durante o período de coletas de dados que aconteceu entre agosto a outubro de 2017. Foram excluídas do estudo mães que tiveram seus filhos internados por outras condições diferentes da prematuridade e mães que se recusarem a participar do estudo por se tratar de uma coleta por meio lúdico.

Para a realização da coleta dos dados foi utilizado um formulário biosociodemográfico elaborado pelos pesquisadores contendo dois blocos, o bloco 1 continha informações da mãe como: nome completo, data de nascimento, idade materna, escolaridade, renda familiar, idade que engravidou, adesão as consultas de pré-natal, quantas consultas realizadas durante a gestação, número de ultrassonografias realizadas, presença de alguma complicação durante a gestação ou no parto, utilização de medicações e tipo de parto, e no bloco 2 continha informações do recém-nascido como: idade gestacional, sexo, Apgar no $1^{\circ}$ e $5^{\circ}$ minuto de vida, peso ao nascimento, diagnóstico de internamento e se tá fazendo uso de algum recurso de oxigenioterapia. 
O projeto foi apreciado e aprovado pelo Comitê de Ética e Pesquisa da Faculdade Independente do Nordeste (CEP/FAINOR), com n n de parecer: 2.234.698 e número de CAAE: 70470717.8.0000.5578. As mães foram convidadas e esclarecidas sobre o objetivo do estudo, as que aceitaram voluntariamente participar do estudo assinaram o termo de consentimento livre e esclarecido (TCLE), corroborando com os fundamentos éticos que se encontram na resolução no 466/12.

Posteriormente, foi aplicado o questionário com dados biosociodemográfico e solicitado após que as mesmas realizassem um desenho representando os sentimentos presentes no momento do internamento de seus filhos. Para este desenho, foi disponibilizado uma folha de papel ofício A4, lápis de cor, caneta hidrográfica, giz de cera, lápis, caneta, borracha e apontador, não foi sugerido o que usar, deixando-a à vontade para realização do desenho. Após a realização do desenho, foi solicitado a mãe que escrevesse uma breve história sobre o desenho, e por fim, dar um título ao mesmo.

Ao final da coleta os dados foram devidamente organizados e analisados. Os dados obtidos a partir do questionário, foram submetidos à uma análise descritiva e apresentados em tabela. As histórias foram transcritas e analisadas a partir da análise do conteúdo temático, descrita por Bardin (2011). A perspectiva de Bardin é composta por um conjunto de técnicas de análise das comunicações, que busca atingir, por meio de procedimentos sistemáticos e objetivos de descrição do conteúdo das mensagens, os indicadores que permitam a inferência de conhecimentos relativos às condições de produção/recepção dessas mensagens.

De acordo com Bardin (2011) essa análise é formada por três fases, sendo a primeira a pré-análise, que consiste na etapa de organização, utilizando procedimentos como: leitura flutuante, formulação de hipóteses e dos objetivos e elaboração de indicadores que fundamentem a interpretação; a segunda fase é a de exploração do material, onde os dados são codificados a partir das unidades de registro; e, a terceira envolve o tratamento dos resultados, inferência e interpretação, etapa na qual se faz a categorização que consiste na classificação dos elementos segundo suas semelhanças e diferenciação, com posterior reagrupamento, em função de características comuns.

Para a identificação das participantes, foi utilizado a letra "M", referentes ao nome mãe e associada a um número, entre 1 a 9 , atribuídos de acordo com a sequência das entrevistas (M1, M2... M9).

As avaliações dos desenhos foram feitas por uma psicóloga, na qual utilizou-se a técnica de House-Tree-Person (HTP), que segundo Xavier (2006), utiliza técnicas de análise projetiva 
e técnicas de ludodiagnóstico aplicadas em indivíduos acima dos 08 anos, consistindo na avaliação projetiva sobre características específicas dos traços, localização e formas de utilização do desenho. Analisando para tanto, a linguagem não verbal e verbal, baseado nos relatos oferecidos pelos consulentes. Segundo Affonso (2009), o ludodiagnóstico utiliza-se de técnicas projetivas para complementar a compreensão dos conflitos expostos pelos indivíduos analisados.

\section{Resultados e discussão}

Os resultados obtidos através da análise desde estudo foram realizados em dois momentos, o primeiro versa sobre a caracterização do perfil das mães, e no segundo momento, discorre sobre a percepção das mães em relação ao internamento de seus filhos sobre o cuidado intensivo neonatal apresentado em duas categorias.

Dentre as 9 mães avaliadas, 55,6\% possuíam o ensino médio completo, não houve predominância para idade materna, ambas categorias apresentaram 33,3\%. Quanto a classificação da renda familiar mensal, a maioria $(66,6)$ das mães relatam receber até 1 salário mínimo, quanto questionadas sobre a adesão para realização de pré-natais, 88,9\% realizaram as consultas, com uma maior média de número de consultas de 1 a 6 (77,8\%). Identificou-se no estudo que $(66,6 \%)$ das mães apresentaram complicação durante o período gestacional, sendo que os mais relatados foram: sangramento vaginal, descolamento prévio de placenta e préeclâmpsia, sendo estas patologias consideradas positiva influência para gestação precoce (Tabela 1).

Tabela 1 - Caracterização de mães de prematuros internados na UTIN. Vitória da Conquista BA, 2017.

\section{$\mathrm{N} \quad(\%)$}

\section{Escolaridade}

Fundamental Incompleto

Fundamental Completo

11,1

Médio Incompleto

11,1

Médio Completo

55,6

Superior

11,1 


\section{Idade}

$<20$

$20-35$

33,3

$>35$

Renda Familiar Mensal

1 salário

2 salários

Adesão ao Pré-natal

Sim

Não

Número de consultas Pré-natal

Nenhuma

1 a 6

7 a 10

Complicações durante a gestação

Sim

$6 \quad 66,6$

Não

Principais complicações relatadas*

Sangramento vaginal

$3 \quad 33,4$

Descolamento Prévio de Placenta

Pré-eclâmpsia

4

22,2

Oligodrâmnio

11,1

*houve mães que apresentaram mais de uma complicação

Fonte: Dados da pesquisa

Afim de responder o objetivo do estudo, qual a percepção das mães em relação ao internamento de seus filhos na UTIN, realizado através da análise do desenho gráfico e das histórias temáticas do conteúdo realizado com mães de prematuros internados nas unidade de cuidado intensivo, emergiram-se 2 categorias: a primeira: Os sentimentos maternos vivenciados durante a hospitalização do filho na UTIN, emergindo as subcategorias: Sentimentos negativos e positivos do processo de internamento; e segunda categorização foi nomeada de espiritualidade e crenças religiosas. 


\section{Os sentimentos maternos vivenciados durante a hospitalização do filho na UTIN}

De acordo com as histórias relatadas pelas mães, observou-se na análise dos resultados do estudo uma chuva de palavras de sentimentos de negatividade, assim como de sentimentos de positividade dentro deste processo.

\subsection{Sentimentos negativos do processo de internamento}

Nesta categoria, segundo os relatos das mães, destacou-se no estudo o processo de insegurança das mesmas após a separação do recém-nascido prematuro, condição esta que leva o bebê a necessidade de cuidados imediatos devido a imaturidade sistêmica. Ao mesmo tempo que as mães sentiam gratidão pela assistência prestado ao filho, muitos eram os sentimentos de medo, angústia, desespero e tristeza, demonstradas nas falas a seguir:

“...na hora que eu sube que seria prematuro o meu coração quais saia pela boca de medo de perde o meu filho..." (M1)

“...bateu um desespero angustia..." (M2)

“...foi muito difícil ela tão pequenina indefesa...” (M4) “...sem muita esperança de vida, foi pra UTI, caso grave...” (M5)

As falas das mães acima descritas, corroboram com o resultado da análise gráfica (representada abaixo), que emergiu sentimentos claros de insegurança, superficialidade, onde a mãe evita o contato naquele momento com seus sentimentos mais hostis

\section{A Vitoriosa}

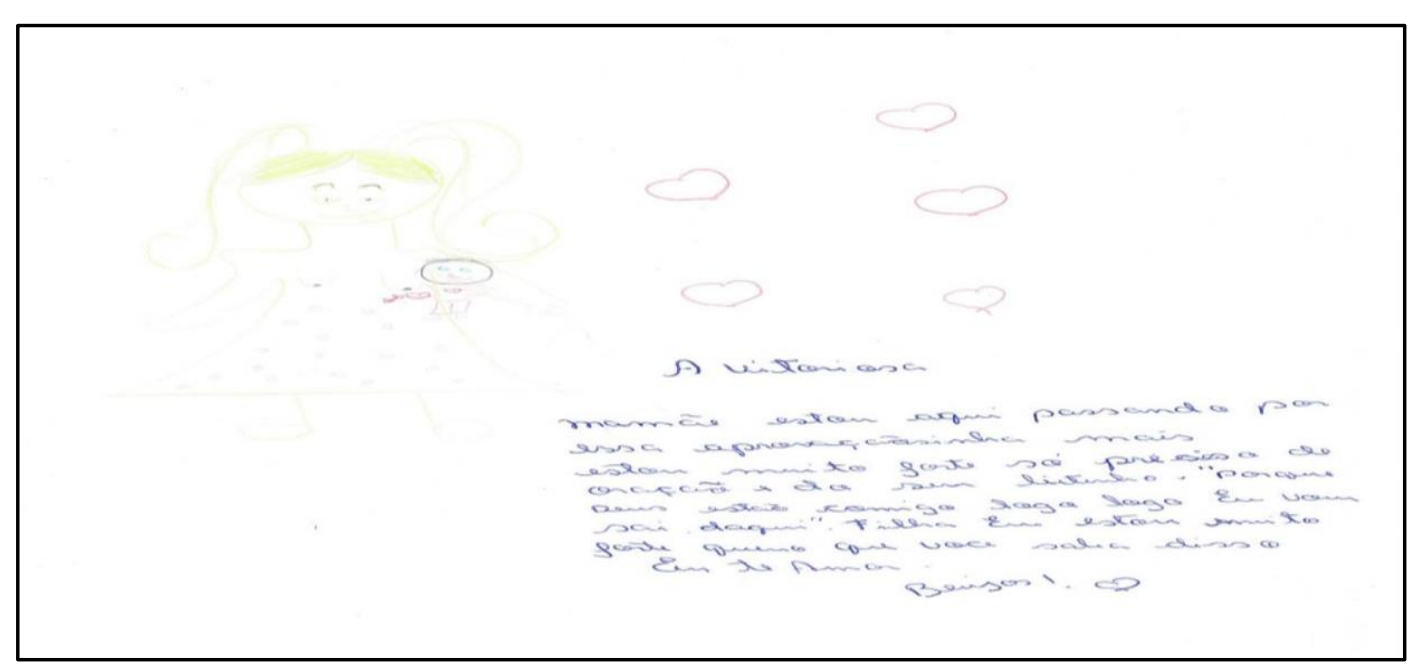

Fonte: Dados da pesquisa 
Segundo Souza e colaboradores (2009), em estudo realizado em um hospital público de referência estadual para gravidez e nascimento de risco no Rio Grande do Norte, com objetivo de conhecer as representações das mães de filhos internados na UTIN, com analise bem similar realizada no presente estudo, encontrou em seus resultados que o nascimento inesperado de um filho prematuro causa reação de choque, tanto pelo nascimento imprevisível, quanto o aspecto indefeso e frágil de um bebê diferente da imagem que foi construída durante o período gestacional. Essa percepção é geradora de sentimento de insegurança e culpa, principalmente para mãe, já que, a mesma não foi capaz de levar a gestação até o final, corroborando assim com os achados do atual estudo.

Veras, Vieira e Morais (2010), justifica que os sentimentos de insegurança e medo que as mães demonstram ao se depararem com a situação crítica de seu filho, geralmente surge a partir do anseio diante do desconhecimento de um futuro incerto e da não possibilidade de retornar com seu filho para casa.

Segundo Fraga e Pedro (2004), o sentimento de culpa por ter um filho prematuro ou que tenha nascido com qualquer outro tipo de patologia, acaba gerando um abalo psicológico à mãe e aos familiares, visto que, as dificuldades em manter um contanto com o filho na UTIN acaba sendo complicado devido uma rotina intensa e regras que a instituição acata para o próprio bem do recém-nascido.

Cruz e colaboradores (2010), discorre ainda em seu estudo que manter o bebê prematuro em uma incubadora aquecida é um cuidado essencial para o seu bom desenvolvimento, porém, acaba comprometendo o contato direto entre mãe e filho devido a uma virtude de aparelhos conectados, sondas, drenos e fios. Em consequência disso, o contato com seus filhos acaba sendo rápido ou muitas vezes inexistentes. Logo, a equipe multidisciplinar atua como mediadora, visto que há uma necessidade do acolhimento e de uma comunicação afetiva e profunda com seus filhos, promovendo uma melhora tanto no ambiente e no estresse diário, bem como, favorecendo na melhora de seus bebês, possibilitando momentos de amor e carinho com seus filhos.

Esses sentimentos de perda, culpa e medo, em suas diversas magnitudes, não devem ser desprezados, mas sim encarados e trabalhados diante ao apoio e diálogo constante entre os profissionais e a família (OLIVEIRA et al., 2013). 


\subsection{Sentimentos positivos do processo de internamento}

Nesta subcategoria emergida segundo análise, as mães mesmo prevalecendo os sentimentos de inseguranças e medos, demonstravam sentimentos de felicidade, esperança e amor com as possíveis evoluções dos bebês. A espera constante do momento em que poderá sair do hospital com seus filhos no colo e clinicamente bem, intensificam seus sentimentos positivos, demonstrados nos relatos a seguir:

“...tava na UTI agora foi pra semi-intensiva, e logo logo pra casa... abençoada feliz e sei $k$ tudo está dando certo meu filho minha vida..." (M2) “...sinto uma felicidade tão grande em sube que logo estaremos em casa...” (M1) "...amor de mãe, asas liberdade..." (M3) "...meu filho, foi um dos presentes melhores..." (M7) “...filha eu estou muito forte quero que você sabia disso. Eu te amo...” (M9)

Corroborante com a análise gráfica abaixo apresentada, foi possível perceber sentimentos que expressavam alegria e amor, similar aos relatos citados. Mesmo que a felicidade apontada, exige uma necessidade de segurança quanto ao futuro do seu filho.

\section{Mamãe Feliz}

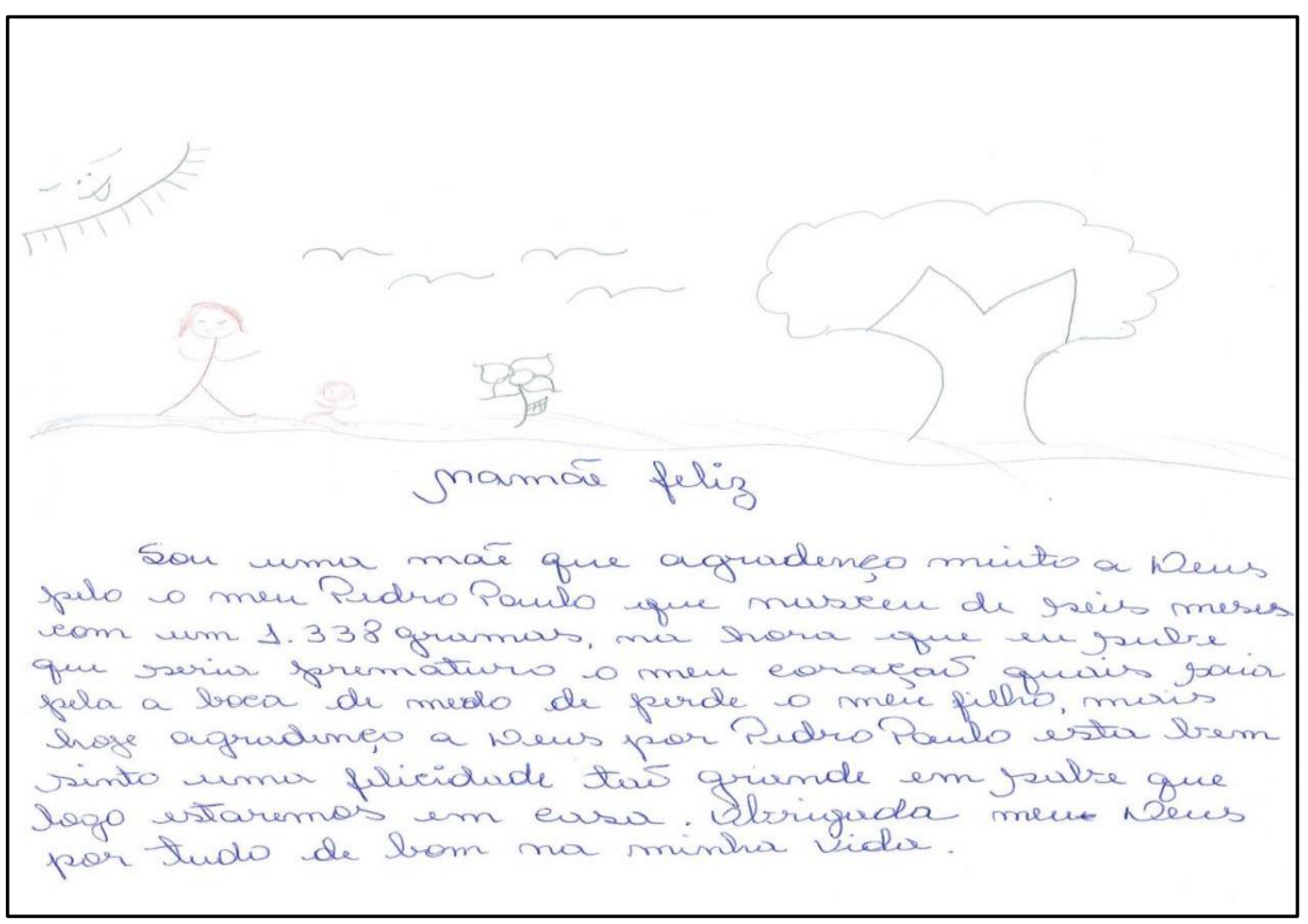

Fonte: Dados da pesquisa 
Sabe-se que a expectativa do nascimento de um filho saudável e perfeito é comum para todos os pais, não seria diferente para as mães participantes desse estudo, visto que, a vontade do cuidar é maior do que os sentimentos negativos.

Souza e colaboradores (2009), relatou na discussão dos seus achados que a mãe mesmo sabendo que o parto será realizado de forma prematura, ela continua mantendo a esperança de que seu filho nasça com uma boa saúde, podendo ainda não separar dela após o nascimento. Ao longo da internação, na medida que o bebê vai apresentando melhoras, a mãe acaba interagindo mais com o seu filho, podendo tocá-lo com mais frequência, pegá-lo no colo por períodos maiores, ocasionando sentimentos de autoconfiança e alegria.

Esse imaginário, quanto a condição de saúde de seus filhos, geralmente tende a ser otimista, causando neles a sensação de que suas forças sobrepujam suas fraquezas. Diante do exposto, um ponto importante a ser considerado para um bom tratamento do bebê, é que exista um bom relacionamento entre os profissionais e os pais, diminuindo o nível de ansiedade e tornado possível a elaboração de estratégias para um bom desenvolvimento de seus filhos (SCHMIDT et al., 2012; DE OLIVEIRA et al., 2013).

\section{Espiritualidade e crenças religiosas}

Esta categoria foi emergida ao perceber a unanimidade segundo as histórias apresentadas através das mães sobre a fé, a religiosidade e a gratidão a Deus, destacado nos resultados deste estudo, de acordo com as falas a seguir:

“...Obrigada meu Deus por tudo de bom na minha vida....” (M1)

“...pedi oração Deus ta abençoado ele..." (M2)

“...Eu comecei a pedir como Deus é lindo e fiel, ele cuidava de tudo...” (M4)

“... a nossa Senhora Aparecida por esse milagre dela já esta ficando boa...” (M6)

"...Hoje em dia graças a Deus estou muito feliz..." (M7)

“...porque Deus estão comigo logo logo Eu vou sai daqui...” (M9)

Condição esta similar ao desenho abaixo, onde a mãe participante aponta Deus como refúgio, esperança e fé nos momentos de maior insegurança e medo. O resultado da análise gráfica demonstra situação de conflito, rigidez emocional e frustação, tendo como difículdade a mãe entrar em contato com as suas próprias emoções. 


\section{Deus é o meu refúgio}

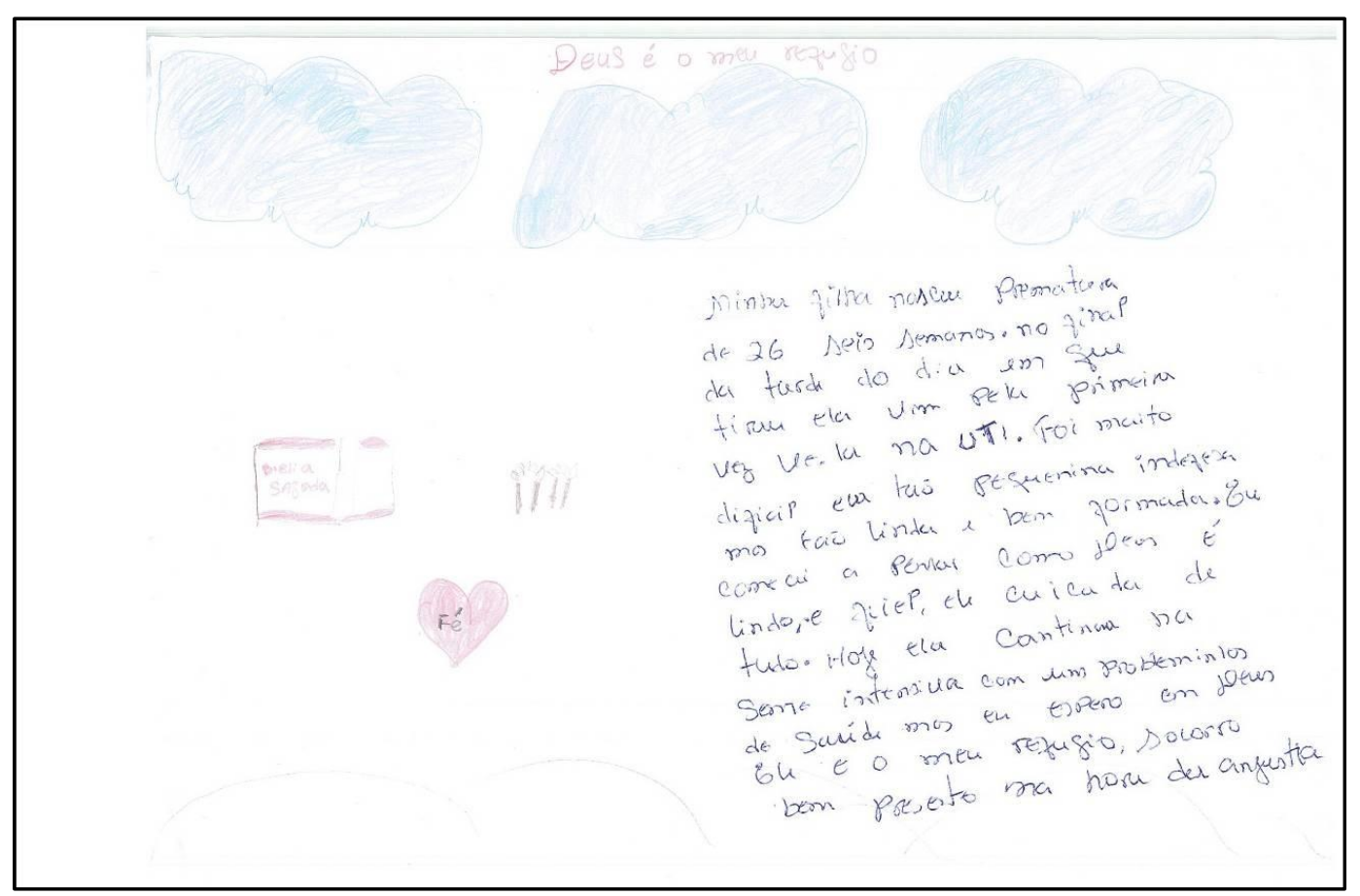

Fonte: Dados da pesquisa

A religiosidade vem sendo destacada como uma das formas mais utilizadas pela população para expressar dimensões emocionais e intuitivas diante de momentos de doenças ou perda de um ente querido. Independentemente de sua crença ou prática religiosa, a espiritualidade surge como um fator marcante na experiência de familiares, sendo referenciada como um suporte para o enfrentamento da angústia decorrente da hospitalização de um parentesco (VÉRAS; VIEIRA; MORAIS, 2010; OLIVEIRA et al., 2013).

Todavia, apesar dos obstáculos, há um sentimento de esperança dessas mulheres sendo os filhos considerados bênçãos ou milagres pelo fato de terem sobrevivido a prematuridade. Mesmo diante da situação, as mesmas já se consideram vencedoras, capazes de sobreviver pela intervenção de Deus (VÉRAS; VIEIRA; MORAIS, 2010).

A fraqueza e o medo diante de um filho hospitalizado geram a necessidade da fé em Deus, uma vez que, torna-se uma tarefa difícil a adaptação e a aceitação de um filho internado na unidade de terapia intensiva neonatal. Diante da tristeza, da angústia e fragilidade condicionadas a hospitalização, a procura por Deus é intensificada no intuito de amenizar o sofrimento e manter a esperança na recuperação de seus filhos (BOTELHO, 2011). 
No presente estudo, foi notável a presença da espiritualidade no dia-a-dia da hospitalização de seus bebês, visto que a fé é considerada como um ponto de suporte, confiança e força para seguir em frente.

A pesquisa em questão apresentou algumas limitações, seria importante incorporar uma avaliação psicológica das mães durante o período de pesquisa, podendo também incluir uma abordagem quantitativa para dispor de dados mais precisos quanto ao perfil sócio demográfico das mães e das crianças, visto que a compreensão de estudos qualitativos é feita de forma mais complexa.

\section{Considerações finais}

Através dessa pesquisa, notou-se que a Unidade de Terapia Intensiva Neonatal emerge sentimentos negativos as mães que vivenciam o processo de internamento, assim como momentos de felicidade, esperança e fé. A importância do cuidado da assistência neonatal deve estender-se às mães visto que são de extrema importante para a sobrevivência de seus filhos.

Portanto, espera-se que este estudo proporcione discussões sobre a importância da humanização no processo de cuidado de recém-nascidos prematuros, afim de contribuir com a melhoria da assistência a esta população, assim como, contribuindo no conhecimento dos estudantes e profissionais da área da saúde.

\section{Referências}

AFFONSO, R.M.L. Ludodiagnóstico: investigação clínica através do brinquedo. Artmed Editora, 2009.

BARDIN, L. Análise de conteúdo. São Paulo: Edições 70; 2011.

BETTIOL, H. et al. Epidemiologia do nascimento pré-termo: tendências atuais. Revista Brasileira de Ginecologia e Obstetrícia, v. 32, n. 2, p. 57-60, 2010.

BOTÊLHO, S. M. Representações sociais de mães sobre prematuros hospitalizados e o cuidar materno. 2011. Tese de Doutorado. Departamento de Saúde, Universidade Estadual do Sudoeste da Bahia, Jequié, BA, 2011. 
DA CRUZ, A.R.M et al. Sentimentos e expectativas da mãe com filho prematuro em ventilação mecânica. Revista Eletrônica de Enfermagem, v. 12, n. 1, 2010.

DA SILVA RABELO, M.Z. et al. Sentimentos e expectativas das mães na alta hospitalar do recém-nascido prematuro. Acta Paul Enferm, v. 20, n. 3, p. 333-7, 2007.

DE OLIVEIRA, K. et al. Vivências de familiares no processo de nascimento e internação de seus filhos em UTI neonatal. Escola Anna Nery Revista de Enfermagem, v. 17, n. 1, p. 4653, 2013.

DOS ANJOS, L.S. et al. Percepções maternas sobre o nascimento de um filho prematuro e cuidados após a alta. Rev Bras Enferm, v. 65, n. 4, p. 571-577, 2012.

FRAGA, I.T.G; PEDRO, E.N.R. Sentimentos das mães de recém-nascidos prematuros: implicações para a enfermagem. Revista gaúcha de enfermagem. Porto Alegre, RS. Vol. 25, n. 1 (abr. 2004), p. 89-97, 2004.

LIMA DE SOUZA, N. et al. Representações de mães sobre hospitalização do filho prematuro. Revista Brasileira de Enfermagem, v. 62, n. 5, 2009.

OLIVEIRA, L.L. et al. Fatores maternos e neonatais relacionados à prematuridade. Revista da Escola de Enfermagem da USP. São Paulo. Vol. 50, n. 3 (maio/jun. 2016), p. 382-389, 2016.

SCHMIDT, K.T. et al. A primeira visita ao filho internado na unidade de terapia intensiva neonatal: percepção dos pais. Escola Anna Nery Revista de Enfermagem, v. 16, n. 1, p. 73 81,2012

VÉRAS, R.M; VIEIRA, J.M.F; MORAIS, F.R.R. A maternidade prematura: o suporte emocional através da fé e religiosidade. Psicol Estud, v. 15, n. 2, p. 325-32, 2010.

XAVIER DA SILVA, M.F; DE VILLEMOR-AMARAL, A.E. A auto-estima no CAT-A e HTP: Estudo de evidência de validade. Avaliação Psicológica, v. 5, n. 2, 2006.

\section{Como citar este artigo (Formato ABNT):}

PALMARELLA NETO, Mauro; SILVA, Valéria G da; DUTRA, Laisla P. Percepção de mães de recémnascidos prematuros sobre o cuidado intensivo neonatal. Id on Line Revista Multidisciplinar e de Psicologia, 2017, vol.11, n.38, p.778-790. ISSN: 1981-1179.

Recebido: 08.11.2017

Aceito: 10.11 .2017 